\title{
A LEFSCHETZ FIXED POINT FORMULA FOR ELLIPTIC DIFFERENTIAL OPERATORS ${ }^{1}$
}

\author{
BY M. F. ATIYAH AND R. BOTT
}

Communicated by E. Spanier, August 20, 1965

Introduction. The classical Lefschetz fixed point formula expresses, under suitable circumstances, the number of fixed points of a continuous map $f: X \rightarrow X$ in terms of the transformation induced by $f$ on the cohomology of $X$. If $X$ is not just a topological space but has some further structure, and if this structure is preserved by $f$, one would expect to be able to refine the Lefschetz formula and to say more about the nature of the fixed points. The purpose of this note is to present such a refinement (Theorem 1) when $X$ is a compact differentiable manifold endowed with an elliptic differential operator (or more generally an elliptic complex). Taking essentially the classical operators of complex and Riemannian geometry we obtain a number of important special cases (Theorems 2,3). The first of these was conjectured to us by Shimura and was proved by Eichler for dimension one.

1. The main theorem. Let $X$ be a smooth compact manifold and let $E, F$ be smooth complex vector bundles over $X$. A differential operator from $E$ to $F$ means a linear map $d: \Gamma(E) \rightarrow \Gamma(F)$ on the spaces of smooth sections which is given in local coordinates by a matrix of partial differential operators with smooth coefficients. By an elliptic complex $E$ on $X$ we mean a sequence $E_{0}, E_{1}, \cdots, E_{n}$ of smooth vector bundles over $X$ and a sequence of differential operators $d_{i}: \Gamma\left(E_{i}\right)$ $\rightarrow \Gamma\left(E_{i+1}\right)$ so that

(i) $d_{i+1} d_{i}=0$ for $i=1, \cdots, n-1$, and

(ii) the sequence of symbols

$$
\cdots \rightarrow E_{i, x} \stackrel{\sigma_{i}(x, \xi)}{\longrightarrow} E_{i+1, x} \rightarrow \cdots
$$

is exact for all $x \in X$ and all nonzero cotangent vectors $\xi$ to $X$ at $x$. Here $\sigma_{i}$ denotes the symbol of $d_{i}$, and $d_{i}$ as well as $\sigma_{i}$ is formally put equal to zero for $i<0$ and $i \geqq n$.

In view of (i) the cohomology groups $H^{i}(E)=$ Kernel $d_{i} /$ Image $d_{i-1}$, are well defined and it is a consequence of (ii) that $H^{i}(E)$ is finitedimensional. Note that, if $n=1$, we are dealing with a single elliptic operator $d_{0} . H^{0}$ is then the space of solutions of $d_{0} u=0$ and $H^{1}$ can

\footnotetext{
1 This work was supported in part by the National Science Foundation.
} 
be shown to be isomorphic to the space of solutions of the adjoint equation $d_{0}^{*} v=0$.

By an endomorphism $T$ of an elliptic complex $E$ we mean a sequence of linear maps $T_{i}: \Gamma\left(E_{i}\right) \rightarrow \Gamma\left(E_{i}\right)$ such that $d_{i} T_{i}=T_{i+1} d_{i}$. Such an endomorphism induces endomorphisms $H^{i} T$ of $H^{i}(E)$ and we define the Lefschetz number of $T$ by the formula

$$
L(T)=\sum_{i=0}^{n}(-1)^{i} \text { Trace } H^{i} T .
$$

For instance if $T$ is the identity endomorphism $I$ the Lefschetz number $L(I)$ is just the Euler-characteristic $\chi(E)=\sum(-1)^{i} \operatorname{dim} H^{i}(E)$, and in particular if $n=1$, so that we are dealing with a single elliptic operator $d_{0}$, then $L(I)=\operatorname{dim} \operatorname{Ker} d_{0}$-dim Coker $d_{0}=$ index $d_{0}$. The problem of computing index $d_{0}$ (and more generally $\chi(E)$ ) has been solved in [1]. In this note we are concerned with endomorphisms which are, in a sense, at the opposite extreme from the identity.

Suppose then that $f: X \rightarrow X$ is a smooth map and that $\phi_{i}: f^{*} E_{i} \rightarrow E_{i}$ are smooth vector bundle homomorphisms. We define linear maps $T_{i}: \Gamma\left(E_{i}\right) \rightarrow \Gamma\left(E_{i}\right)$ as the composition

$$
\Gamma\left(E_{i}\right) \stackrel{f^{*}}{\rightarrow} \Gamma\left(f^{*} E_{i}\right) \stackrel{\phi_{i}}{\rightarrow} \Gamma\left(E_{i}\right)
$$

If further $d_{i} T_{i}=T_{i+1} d_{i}$ then the $T_{i}$ define an endomorphism $T$ of the elliptic complex $E$. An endomorphism of this type, associated to $\left\{f, \phi_{i}\right\}$ we call a geometric endomorphism of $E$.

Finally we define a fixed point $P$ of a map $f: X \rightarrow X$ to be simple if $\operatorname{det}\left(1-d f_{P}\right) \neq 0$, where $d f_{P}$ is the induced map on the tangent space to $X$ at $P$. This implies that $P$ is an isolated fixed point. Hence if all fixed points of $f$ are simple it follows, since $X$ is compact, that they are finite in number. Let us observe that $\phi_{i}$ may be interpreted as a family of linear maps $\phi_{i, P}: E_{i, f(P)} \rightarrow E_{i, P}$, parameterized by $P \in X$. Hence if $P$ is a fixed point, $f(P)=P$, then $\phi_{i, P}$ is an endomorphism of the vector space $E_{i, P}$ and so Trace $\phi_{i, P}$ is defined.

TheOREM 1. Let $E$ be an elliptic complex on $X$ and let $T$ be a geometric endomorphism of $E$ associated to $\left(f, \phi_{i}\right)$ where $f: X \rightarrow X$ has only simple fixed points. Then the Lefschetz number $L(T)$ is given by the formula

$$
L(T)=\sum_{P} \nu(P)
$$

where the summation is over the set of fixed points of $f$ and $\nu(P)$ is given by 


$$
\nu(P)=\frac{\sum(-1)^{i} \operatorname{Trace} \phi_{i, P}}{\left|\operatorname{det}\left(1-d f_{P}\right)\right|}
$$

REMARKS.

(1) Note that the formula for $\nu(P)$ does not explicitly involve the differential operators $d_{i}$. This is in marked contrast to the index formula [1, Theorem 1]. Of course the $d_{i}$ are implicitly involved by the condition $T_{i} d_{i}=T_{i+1} d_{i}$.

(2) If we take $E$ to be the de Rham complex (exterior differential forms with the exterior derivative), and $\phi_{i}$ to be the natural map on $i$-forms induced by $f$, we find

$$
\nu(P)=\frac{\operatorname{det}\left(1-d f_{P}\right)}{\left|\operatorname{det}\left(1-d f_{P}\right)\right|}= \pm 1
$$

and we recover the original Lefschetz theorem, since $L(T)$ is now the usual Lefschetz number of the map $f$.

(3) Note that in general $\nu(P)$ is a complex number and not an integer. The classical Lefschetz formula, where $\nu(P)= \pm 1$, is highly special in this direction. Note on the other hand that the Lefschetz number $L(T)$ is a linear combination of traces and so, if $T$ is of finite order, $L(T)$ will be an algebraic integer. In these cases Theorem 1 leads to "integrality theorems" analogous to the integrality theorems obtainable from the index theorem.

2. The holomorphic case. Using the $\bar{\partial}$-complex on a complex manifold and the Dolbeault isomorphism Theorem 1 leads easily to:

Theorem 2. Let $X$ be a compact complex manifold, $V$ a holomorphic vector bundle over $X, f: X \rightarrow X$ a holomorphic map with simple fixed points and $\phi: f^{*} V \rightarrow V$ a holomorphic vector bundle homomorphism. Let $H^{i}(f, \phi)$ denote the composite homomorphism

$$
H^{i}(X, v) \stackrel{f^{*}}{\longrightarrow} H^{i}\left(X, f^{*} v\right) \stackrel{\phi^{*}}{\longrightarrow} H^{i}(X, v)
$$

where $V$ is the sheaf of germs of holomorphic sections of $V$. Then

$$
\sum(-1)^{i} \text { Trace } H^{i}(f, \phi)=\sum_{P} \nu(P)
$$

where the summation is over the fixed points of $f$,

$$
\nu(P)=\frac{\text { Trace } \phi_{P}}{\operatorname{det}_{C}\left(1-d f_{P}\right)}
$$


and $d f_{P}$ denotes the $\mathrm{C}$-endomorphism ${ }^{2}$ of the tangent space to $X$ at $P$ induced by $f$.

Like the Riemann-Roch theorem, Theorem 2 makes sense and is also true in the case of abstract algebraic geometry. As a simple corollary of Theorem 2 we have:

CoRollary 1. Any rational projective algebraic manifold has the fixed point property for holomorphic maps.

3. The Riemannian case. Let $X$ be a compact oriented Riemann manifold of dimension $2 l$. Let $H^{l}$ denote the space of (complexvalued) harmonic $l$-forms. The usual dualizing operator $*: H^{l} \rightarrow H^{l}$ has $(*)^{2}=(-1)^{l}$. Hence $\alpha=i^{l^{2}} *$ has $\alpha^{2}=1$. Let $H_{+}^{l}$ and $H_{-}^{l}$ denote the $+1,-1$ eigenspaces of $\alpha$. Now there is an elliptic operator (cf. [1, (3.1)(ii) ]), canonically associated to the structure of $X$, whose index is $\operatorname{dim} H_{+}^{l}-\operatorname{dim} H_{-}^{l}$. If $l$ is even this is the Hirzebruch index of $X$. More generally therefore if $f: X \rightarrow X$ is an oriented isometry we may define the Hirzebruch number

$$
\tau(f)=\operatorname{Trace} f_{+}^{l}-\operatorname{Trace} f_{-}^{l}
$$

where $f_{+}^{l}$ is the endomorphism of $H_{+}^{l}$ induced by $f$ and similarly for $f_{-}^{l}$. It is a special Lefschetz number. Its interest lies in the fact that it depends only on the section of $f$ on the cohomology of $X$ and so it is quite easily computed. Applying Theorem 1 to this special operator we obtain

THEOREM 3. Let $X$ be a compact oriented smooth manifold of dimension 2l. Let $f: X \rightarrow X$ be an oriented isometry with isolated fixed points. For each fixed point $P$ let $T_{P}=\bigoplus_{k=1}^{l} T_{P, k}$ be a decomposition of the oriented tangent space $T_{P}$ into oriented 2-planes $T_{P, k}$ invariant under $d f_{P}$, and let $d f_{P}$ induce a rotation through an angle $\theta_{P, k}$ on $T_{P, k}$. Then the Hirzebruch number $\tau(f)$ is given by the formula

$$
\tau(f)=\sum_{P} \nu(P)
$$

where

$$
\nu(P)=i^{l} \prod_{k=1}^{l} \cot \left(\frac{\theta_{P, k}}{2}\right)
$$

${ }^{2}$ For a complex linear endomorphism we must distinguish carefully between detc and $\operatorname{det}_{R}$ the determinant of the underlying real transformation: they are related by $\operatorname{det}_{R}=|\operatorname{det} C|^{2}$. 
If $G$ is a compact Lie group acting differentiably on $X$ then by averaging over $G$ we can find a Riemannian metric invariant under $G$. Theorem 3 can then be used to obtain results concerning such group actions. For example Theorem 3 together with some delicate number theory (for which we are indebted to J. Milnor) gives:

CoRollary 2. Let $G$ be a compact Lie group acting differentiably on $a$ (homology) sphere. Assume that $G$ has just two fixed points $P, Q$ and that elsewhere the action is free. Then the representations of $G$ on the tangent spaces at $P, Q$ are equivalent.

This result is particularly interesting when $G$ is a finite cyclic group since it implies that two lens spaces which are $h$-cobordant are isometric (cf. $[3, \S 12]$ ).

4. Outline of proof. If $T$ is a geometric endomorphism of an elliptic complex $E$ the operators $T_{i}: \Gamma\left(E_{i}\right) \rightarrow \Gamma\left(E_{i}\right)$ do not unfortunately have traces in any accepted functional analytic sense. If, however, $T$ is defined by a map $f$ with only simple fixed points it turns out that one can define a trace for $T_{i}$ which we call the flat trace and denote bv Trace $^{b} T_{i}$. It is defined as a limit

$$
\operatorname{Trace}^{b} T_{i}=\lim _{n \rightarrow \infty} \operatorname{Trace}\left(T_{i} \circ I_{n}\right)
$$

where $I_{n}$ is a sequence of operators with smooth kernels which approximate the identity operator in a certain rather precise way (referred to as flat approximation). Note that the composition $T_{i} \circ I_{n}$ has smooth kernel and therefore has a trace in an accepted sense, namely as $\int k(x, x) d x$, where $k(x, y)$ is the kernel.

From its definition as the limit of a sequence of integrals it is not difficult to evaluate Trace ${ }^{b} T_{i}$ explicitly, and we obtain

$$
\operatorname{Trace}^{\natural} T_{\imath}=\sum_{P} \frac{\text { Trace } \phi_{i, P}}{\left|\operatorname{det}\left(1-d f_{P}\right)\right|}
$$

where the summation is over the fixed points of $f$. To prove Theorem 1 therefore, we have to show that

$$
\sum(-1)^{i} \text { Trace } T_{i}=\sum(-1)^{i} \operatorname{Trace} H^{i} T .
$$

For this purpose consider the "Zeta-function" [4]

$$
\zeta_{i}(s)=\operatorname{Trace}\left\{(1+\Delta)^{-s} \circ T_{i}\right\}
$$


where $\Delta$ is the operator ${ }^{3} d d^{*}+d^{*} d$ relative to Riemannian structures on the $E_{i}$ and $X$. For $\operatorname{Re}(s)$ large this trace exists in the Hilbert Space sense and there, by the Hodge theory:

$$
\sum(-1)^{i} \zeta_{i}(s)=\sum(-1)^{i} \text { Trace } H^{i} T .
$$

Using more delicate properties of $\Delta$, as given in [2], and more recently further refined by Hormander and Seeley, one then shows that Trace $^{b}\left\{(1+\Delta)^{-8} \circ T_{i}\right\}$ defined as in (1), furnishes an analytic continuation of $\zeta_{i}(s)$ to the whole plane. The result then follows by putting $s=0$.

\section{REFERENCES}

1. M. F. Atiyah and I. M. Singer, The index of elliptic operators on compact manifolds, Bull. Amer. Math. Soc. 69 (1963), 422-433.

2. J. Kotake and M. Narasimhan, Regularity theorems for fractional powers of a linear elliptic operator, Bull. Soc. Math. France 90 (1962), 449-471.

3. J. Milnor, Whitehead torsion, (to appear).

4. S. Minakshisundaram and A. Pleijel, Some properties of the eigenfunctions of the Laplace-operator on Riemann manifolds, Canad. J. Math. 1 (1949), 242-256.

OXFORD UNIVERSITY, HARVARD UNIVERSITY.

${ }^{3}$ For simplicity of exposition we assume all $d_{i}$ are of the same order. 